\title{
Aneurysmal subarachnoid hemorrhage: long-term trends in incidence and survival in Olmsted County, Minnesota
}

\author{
Enrico Giordan, MD, ${ }^{1}$ Christopher S. Graffeo, MD, MS, ${ }^{1}$ Alejandro A. Rabinstein, MD, ${ }^{2}$ \\ Robert D. Brown Jr., MD, MPH, ${ }^{1}$ Walter A. Rocca, MD, MPH, ${ }^{2,3}$ Alanna M. Chamberlain, $\mathrm{PhD},{ }^{3}$ and \\ Giuseppe Lanzino, MD'
}

\begin{abstract}
1Department of Neurologic Surgery, and 'Division of Epidemiology, Department of Health Sciences Research, Mayo Clinic; and 2Department of Neurology, Mayo Clinic College of Graduate Medical Education, Rochester, Minnesota
\end{abstract}

\begin{abstract}
OBJECTIVE Recent population-based and hospital cohort studies have reported a decreasing incidence of aneurysmal subarachnoid hemorrhage (aSAH) and declining aSAH-associated case-fatality rates. Principal drivers of these trends are debated, but improvements in smoking cessation and hypertension control may be critical factors.

METHODS The population-based medical records linkage system of the Rochester Epidemiological Project was used to document aSAH incidence and 30-day case fatality rates during a 20-year study period (1996-2016) in Olmsted County, Minnesota. Incidence rates in the study period were compared with data from a previous Olmsted County study concerning aSAH incidence from 1965 to 1995 and with regional trends in tobacco use.

RESULTS One hundred nineteen incident cases of aSAH were included. The median age at hemorrhage was 59 years (range 16-94 years), and 74 patients were female (62.2\%). The overall average annual aSAH incidence rate was 4.2/100,000 person-years (P-Y). The aSAH incidence rate decreased from 5.7/100,000 in 1996 to 3.5/100,000 P-Y in 2011-2016. The overall aSAH-associated 30 -day case-fatality rate was $21.9 \%$ and declined by approximately $0.5 \%$ annually. An accelerated decline in the fatality rate (0.9\%/year) was observed from 2006-2016. Smoking among adult Olmsted County residents decreased from $20.4 \%$ in 2000 to $9.1 \%$ in 2018 .

CONCLUSIONS A decline in the incidence of aSAH and 30-day case-fatality rate from 1996 to 2016 was observed, as well as an accelerated decline of the fatality rate from 2006 to 2016 . These findings confirm and extend the trends reported by prior studies in the same population. The decrease in aSAH in the years studied paralleled a noticeable reduction in the population smoking rates.
\end{abstract}

https://thejns.org/doi/abs/10.3171/2019.12.JNS192468

KEYWORDS aneurysm; subarachnoid hemorrhage; incidence; epidemiology; survival; smoke; vascular disorders

$\mathrm{T}$ HE incidence of aneurysmal subarachnoid hemorrhage (aSAH) varies in different regions of the world and across ethnic groups. ${ }^{3}$ Recent population-based studies have reported a decreasing incidence of aSAH. ${ }^{3,9}$ Both population-based cohorts ${ }^{3,8}$ and hospitalbased cohorts ${ }^{9}$ have also suggested improved survival after the hemorrhage. Lower mortality may be related to a better understanding of the disease and its complications, advances in neurocritical care, and the increased use of endovascular therapy to treat ruptured aneurysms. ${ }^{9}$ However, the reasons underlying the reduction in the incidence of aSAH are a matter of debate. Recent studies have associated the decline in aSAH rates with the decline of smoking rates and, to a lesser degree, to the better treatment of hypertension, the two most influential modifiable risk factors for formation, growth, and possible rupture of intracranial aneurysms (IAs). ${ }^{3,5,8}$ The Rochester Epidemiological Project (REP) is a population-based medical records linkage system incorporating virtually all residents of Olmsted County, Minnesota, which provides a unique opportunity to evaluate incidence and trends of aSAH in a relatively homogeneous North American population..$^{14}$ In this study, we report overall and age- and sex-specific incidence rates, and 30-day case fatality rates of aSAH over 20 years in Olmsted County. We also compare our trends for aSAH with regional trends in smoking habits.

ABBREVIATIONS $A C A=$ anterior cerebral artery; $\mathrm{ACOA}=$ anterior communicating artery; $\mathrm{aSAH}=$ aneurysmal subarachnoid hemorrhage; $\mathrm{Cl}=\mathrm{confidence} \mathrm{interval;} \mathrm{IA}=$ intracranial aneurysm; ICA = internal carotid artery; $M C A=$ middle cerebral artery; $O R=$ odds ratio; $P-Y=$ person-years; REP = Rochester Epidemiological Project; $u I A=$ unruptured IA.

SUBMITTED September 8, 2019. ACCEPTED December 6, 2019.

INCLUDE WHEN CITING Published online February 21, 2020; DOI: 10.3171/2019.12.JNS192468. 


\section{Methods \\ Data Source}

The REP is a population-based medical records linkage system incorporating patient data from all local clinical practices in Olmsted County as described elsewhere. As a result, the REP essentially captures and maintains a repository of all ongoing medical data pertinent to the care of Olmsted County residents, with greater than $95 \%$ participation. Details regarding the linkage system, which is updated frequently, as well as testing of its reliability and validity, and protocols for data indexing and abstraction, have been previously described in detail. ${ }^{14}$

In the present analysis, we utilized the REP resources to identify all patients diagnosed with aSAH during the 20-year study period (1996-2016). The assessment of intracranial hemorrhage using the REP codes was described in a previous study. ${ }^{12}$ Patients with an incident hemorrhage were followed periodically for 2 years after the acute event. The primary search found 1326 Olmsted County residents with any type of intracranial hemorrhage during the years of study. Comprehensive medical record review was completed, inclusive of all inpatient and outpatient clinical records, computerized indices of medical diagnosis and surgical procedure codes, medical billing records, death certificates, and autopsy reports. The complete review was conducted to avoid missing any aSAH cases that may have been mislabeled as non-aSAH intracranial hemorrhage.

\section{Study Criteria}

Olmsted County residents with incident cases of ruptured saccular IAs occurring from 1996 to 2016 were included. The presence of a saccular IA was confirmed for all cases based on the review of imaging studies, operative notes, or autopsy reports. IAs morphologically classified as fusiform and/or dissecting aneurysms were excluded. IAs arising distal to the circle of Willis in patients with concomitant acute bacterial endocarditis, systemic sepsis, or bacteremia were excluded. Patients without confirmed IAs were excluded. Individuals who moved into the county to facilitate diagnosis and treatment of a known or suspected aSAH were also excluded.

\section{Data Collection}

The study was approved by the IRB of our institution (Mayo Clinic), and only patients who gave signed consent for the utilization of their information for research purposes were included. An experienced nurse abstractor reviewed the complete inpatient and outpatient medical records of all 1326 patients identified with an intracranial hemorrhage on initial REP query. All patients positively screened by the abstractor were secondarily reviewed by a single study investigator (E.G.), who confirmed the aSAH diagnosis. Autopsy reports documenting death by cerebral hemorrhage were screened as well, and those cases with a medical history and pathological findings positive for aSAH were reviewed. Autopsy report screening was performed to avoid underestimating case fatality rates. For patients meeting the study criteria, the study investigator also recorded all pertinent clinical information. Data abstracted included: patient sex, age, race, and the date of
aSAH; aneurysm location, size, morphology, and treatments; medical history, including hypertension (defined as either a documented diagnosis in the medical record by a treating physician, or the outpatient use of oral antihypertensive medications) and history of smoking (defined as current, former with no smoking for $>12$ months, or never smoker); family history of IA/aSAH; active antiplatelet/ anticoagulant therapy at time of diagnosis; and degree of aSAH as defined by Fisher scale grades. ${ }^{4}$

IA size was measured using the maximal diameter, and modeled as both a continuous and categorical variable, using the thresholds $<10 \mathrm{~mm}$ (small), 10-25 $\mathrm{mm}$ (large), and $>25 \mathrm{~mm}$ (giant). If no radiograph or direct report was available for size confirmation, the patient was omitted (missing data). IA location was categorically modeled using a 2-tiered stratification that identified the parent vessel and that vessel's anterior/posterior circulation categorization: aneurysms arising from the middle cerebral artery (MCA), anterior communicating artery (ACoA), anterior cerebral artery (ACA), internal carotid artery (ICA), ophthalmic artery, posterior communicating artery, or anterior choroidal artery were considered anterior circulation, whereas those arising from the basilar artery, vertebral artery, anterior/posterior inferior cerebellar arteries, or posterior cerebral artery were classified as in the posterior circulation.

Case fatality rates from aSAH were captured as a dichotomous endpoint, with time-to-event data recorded from diagnosis to death or last follow-up (specified as clinical, radiographic, or both). To analyze trends over 50 years, aSAH incidence rates were combined with data from the study by Menghini and colleagues, ${ }^{12}$ which utilized almost the same methodology and reported data in the same population from 1965 to 1995.

Detailed data regarding the frequency of smoking among the adult population of Olmsted County were available and provided for this study in 2000 and $2018^{10}$ ("US Centers for Disease Control and Prevention. Best Practices for Comprehensive Tobacco Control Programs 2014"). The demographic characteristics in 2010 (the census year closest to the midpoint of the study interval) for Olmsted County, Minnesota, indicated that $85.7 \%$ of the population was Caucasian (compared with $72.4 \%$ of the US population in the same year) and $51 \%$ female. Education among adult patients included $94.3 \%$ with a high school diploma or higher education (compared with $85 \%$ in the US). The percentage of families with incomes less than the poverty level was $8.5 \%$, versus $15.3 \%$ for the entire US population (https://www.census.gov).

\section{Statistical Analysis}

Descriptive statistics were reported as median and range for continuous variables, and proportion with a percentage for categorical variables. Incidence rates per 100,000 population per year were calculated for aSAH overall and in age and sex strata. Overall incidence rates were standardized using the US 2010 census data for citizens self-reporting Caucasian ethnicity, given the predominance of this group in Olmsted County. The population at risk was estimated using census data for 1990 , 2000, and 2010, with linear interpolation for intercensus 
TABLE 1. A summary of aSAH patient characteristics

\begin{tabular}{|c|c|}
\hline Characteristic & Value \\
\hline No. of patients w/ aSAH & 119 \\
\hline Median age at diagnosis (range), yrs & $59(16-94)$ \\
\hline \multicolumn{2}{|l|}{ Race $(\%)$} \\
\hline Caucasian & 84 \\
\hline Black or African American & 5.9 \\
\hline Asian or Pacific Islander & 2.5 \\
\hline Hispanic or Latino & 3.4 \\
\hline Unknown & 4.2 \\
\hline Female sex, n (\%) & $74(62.2)$ \\
\hline Median IA size (range), mm & $6(2-25)$ \\
\hline Multiple aneurysms, n (\%) & $19(16.0)$ \\
\hline \multicolumn{2}{|l|}{ Location (\%) } \\
\hline Paraclinoid ICA & 4.8 \\
\hline PCoA & 18.3 \\
\hline Supraclinoid ICA & 7.7 \\
\hline $\mathrm{ACA}$ (including $\mathrm{ACOA}$ ) & 39.4 \\
\hline MCA & 15.4 \\
\hline Posterior circulation & 14.4 \\
\hline \multicolumn{2}{|l|}{ Cigarette smoking (\%) } \\
\hline No & 42.7 \\
\hline Current smoker & 40.8 \\
\hline Quit (>12 mos) & 16.5 \\
\hline Family history of aSAH (\%) & 9.5 \\
\hline Hypertension (\%) & 48.6 \\
\hline
\end{tabular}

years, as described by Gupta. ${ }^{6}$ Age- and sex-specific incidence rates were calculated overall and for 5-year intervals spanning the study period (1996-2016). Confidence intervals (CIs) were estimated via the Poisson distribution, and Poisson regression was used to assess the relationship between crude incidence rates and year of diagnosis, age at diagnosis, sex, and aneurysm size. All statistical tests were 2 -sided, the type I error rate was 0.05 , and analyses were conducted using Stata (version 13.0, StataCorp). Any data not published within the article are available from the corresponding author upon reasonable request.

\section{Results}

During the study period, 196 cases of subarachnoid hemorrhage were identified. After excluding 32 patients because no aneurysm was identified ("sine materia") and 45 patients in whom the hemorrhage was related to an underlying arteriovenous malformation or dural arteriovenous fistula, 119 patients were confirmed to have suffered an aSAH. Five patients were identified as having suffered an aSAH based on autopsy.

The median age at the aSAH was 59 years (range 16-94 years), and there was a female predominance ( 74 females, 45 males; female/male ratio 1.64:1). Demographics, aneurysm characteristics, and clinical presentation of patients with aSAH are summarized in Table 1 . The overall annual incidence of aSAH was 4.2/100,000 person-years (P-Y). The crude incidence rate decreased from 5.7/100,000 P-Y in 1996-2000 to 3.5/100,000 P-Y in 2011-2016. A summary of aSAH incidence is described in Tables 2 and 3.

The average overall 30-day case-fatality rate from aSAH was $21.9 \%$, and the rate dropped by $0.5 \%$ per year from 1996 to 2016 . The decline in case fatality accelerated to $0.9 \%$ per year from 2006 to 2016 . The overall trends of the incidence of aSAH and case-fatality rates over 5-year intervals are as illustrated in Figs. 1 and 2. The prevalence of smoking among the adult population ( $>18$ years old) in Olmsted County decreased from $20.4 \%$ in 2000 to $9.1 \%$ in 2018.

After combining our data with the results from the study of Menghini et al., ${ }^{12}$ and compounding the effects over 10-year intervals from 1965 to 2016, the aSAH incidence rates steadily decreased from 8.7 to $3.5 / 100,000 \mathrm{P}-\mathrm{Y}$ $(\mathrm{p}<0.05)$ over the 50 years (Fig. 1).

The proportion of patients with aSAH and a confirmed diagnosis of arterial hypertension increased from $42.9 \%$ (1996-2000) to 58.6\% (2011-2016), while the proportion of patients with aSAH who were active smokers decreased from 47.3\% (1996-2000) to 42.3\% (2011-2016; Table 4).

We did not find any significant association between smoking habits (odds ratio [OR] 1.06, 95\% CI 0.55-2.05, $\mathrm{p}=0.875)$ or positive medical history of hypertension (OR $0.71,95 \%$ CI $0.30-1.68, p=0.435$ ) and survival of patients with aSAH, after having adjusted for sex, age, location, and aneurysm size.

\section{Discussion}

In a population-based study of a relatively homogeneous North American cohort from 1996 to 2016, we found the average incidence of aSAH to be $4.2 / 100,000 \mathrm{P}-\mathrm{Y}$. The 30 -day case-fatality rate was approximately $22 \%$. Both incidence and mortality showed a trend toward decreasing rates in this 20-year interval, which continued a similar trend previously noted in the same population. ${ }^{11,12}$

TABLE 2. Five-year incidence rates of aSAH in Olmsted County, Minnesota (1996-2016)

\begin{tabular}{lcccc}
\hline Variable & $1996-2000$ & $2001-2005$ & $2006-2010$ & $2011-2016$ \\
\hline Male & 3.6 & 4.1 & 2.4 & 5.3 \\
\hline Female & 7.7 & 5.6 & 3.4 & 1.6 \\
\hline Total & 5.7 & 4.8 & 2.9 & 3.5 \\
\hline Adjusted* & 5.6 & 4.8 & 2.98 & 3.58 \\
\hline
\end{tabular}

* Age- and sex-adjusted incidence rates per 100,000 P-Y adjusted to the 2010 US Caucasian population. 
TABLE 3. Average annual incidence rates of aSAH in Olmsted County, Minnesota (1996-2016), by age group

\begin{tabular}{lccccccc}
\hline & \multicolumn{7}{c}{ Incidence Rates (no. of patients) } \\
\cline { 2 - 8 } aSAH Cases & $0-34$ & $35-44$ & $45-54$ & $55-64$ & $65-74$ & $75-84$ & $85+$ \\
\hline Men & $0.3(3)$ & $3.9(8)$ & $8.9(17)$ & $8.4(11)$ & $6.3(5)$ & $2.2(1)$ & $6.8(1)$ \\
\hline Women & $0.6(4)$ & $3.6(8)$ & $11.3(23)$ & $8.5(12)$ & $13.1(12)$ & $17.5(10)$ & $15.6(5)$ \\
\hline Total & $0.4(7)$ & $3.8(16)$ & $10.2(40)$ & $8.5(23)$ & $9.9(17)$ & $10.6(11)$ & $12.9(6)$ \\
\hline
\end{tabular}

The REP provides a unique opportunity to analyze trends in the incidence and 30-day fatality rates of aSAH. ${ }^{11-13}$ In a study encompassing the years 1945 until 1974 in Olmsted County, Phillips and coworkers reported the incidence of SAH to be fairly constant at 11 per 100,000 P-Y. ${ }^{13}$ A major limitation of that study was the lack of neurological imaging at the time; only $70 \%$ of patients with SAH had an aneurysm confirmed either by angiography or autopsy. In a subsequent epidemiological study by Brown and colleagues extending to 1989 , a nonsignificant trend toward decreasing incidence of SAH was noted both in men and women in the same population. ${ }^{1}$ A more recent report by Menghini and coworkers on the incidence of aSAH in Olmsted County provided a better comparison because they utilized the same methodology used in the present study and included only patients with documented (either by angiography or autopsy) ruptured aneurysms. ${ }^{12}$ Menghini and coworkers reported an overall incidence of aSAH of 6.9/100,000 P-Y in the 30 years from 1965 to $1995 .^{12}$ When stratified by decade, the overall incidence decreased from 8.7 in 1965-1974, to 7.2 in 1975-1984, to 5.6/100,000 $\mathrm{P}-\mathrm{Y}$ in 1985-1995. Our study confirms a further decrease over the ensuing 20 years, reaching 3.5/100,000 P-Y in the 2006-2016 decade (Fig. 1).

Similar trends have been observed in other studies of different populations. In the Finnish population, which has traditionally been considered to have a higher incidence of aSAH compared to other populations, there was a $24 \%$ reduction in the incidence of aSAH from 11.7/100,000 P-Y in $1995-2000$ to $8.9 / 100,000$ P-Y in $2010-2012 .{ }^{8}$ The

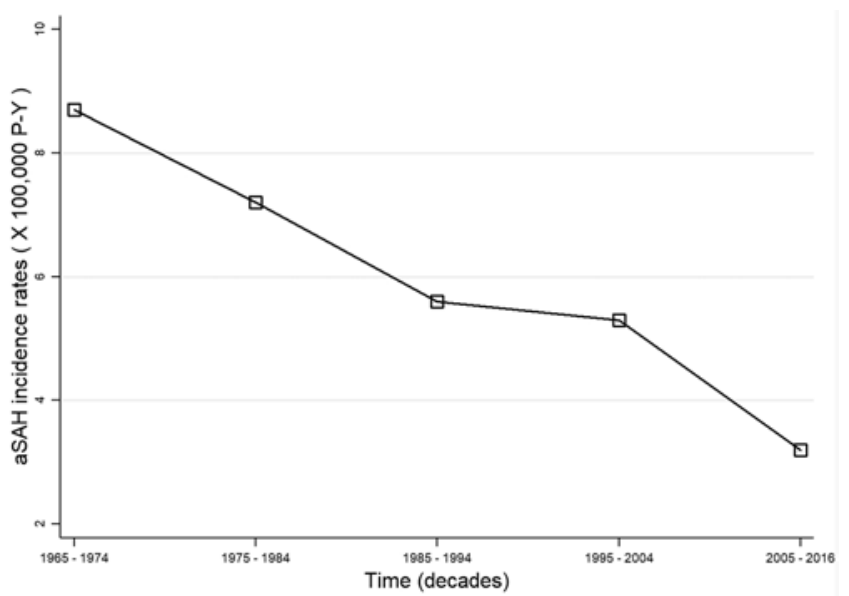

FIG. 1. Decline of aSAH incidence rates by decade from 1965 to 2016 in Olmsted County, Minnesota. crude incidence rates preferentially decreased in women younger than 50 years and were associated with a reduction in daily smoking in the same population. ${ }^{8}$ In a recent meta-analysis, global SAH incidence declined from $10.2 / 100,000 \mathrm{P}-\mathrm{Y}$ in 1980 to $6.1 / 100,000 \mathrm{P}-\mathrm{Y}$ in $2010 .^{3} \mathrm{In}$ this same study, it was documented that the global SAH incidence declined by $7.1 \%$ with every millimeter of mercury decrease in systolic blood pressure and by $2.4 \%$ with every percentage point decrease in smoking prevalence. ${ }^{3}$ In addition to decreasing rates of smoking and better control of hypertension, other factors may contribute to a lower incidence of aSAH in developed countries. These factors include the increased detection rate and prophylactic treatment of unruptured IAs (uIAs) and other possible pharmacological interactions with aneurysm formation, growth, and rupture. ${ }^{2}$ Indeed, several studies have suggested a protective role of aspirin against aneurysm rupture.?

In parallel with decreasing rates of aSAH, trends in Olmsted County suggest a progressive improvement in 30-day fatality rates. In this population, the overall casefatality rate at 30 days was $58 \%$ in the period between 1945 and 1974 and the rate dropped by $0.5 \%$ per year from 1996 to 2016, and by $0.9 \%$ per year from 2006 to 2016 . These figures are staggering if one considers that patients with non-aSAH (who tend to have a better outcome than patients with aSAH) were excluded. In the same population, 30-day case-fatality rates for SAH were better but still quite high between 1985 and 1989 (43\% in men and $33 \%$ in women). Our study shows that these figures have continued to improve; the 30-day case-fatality rate for

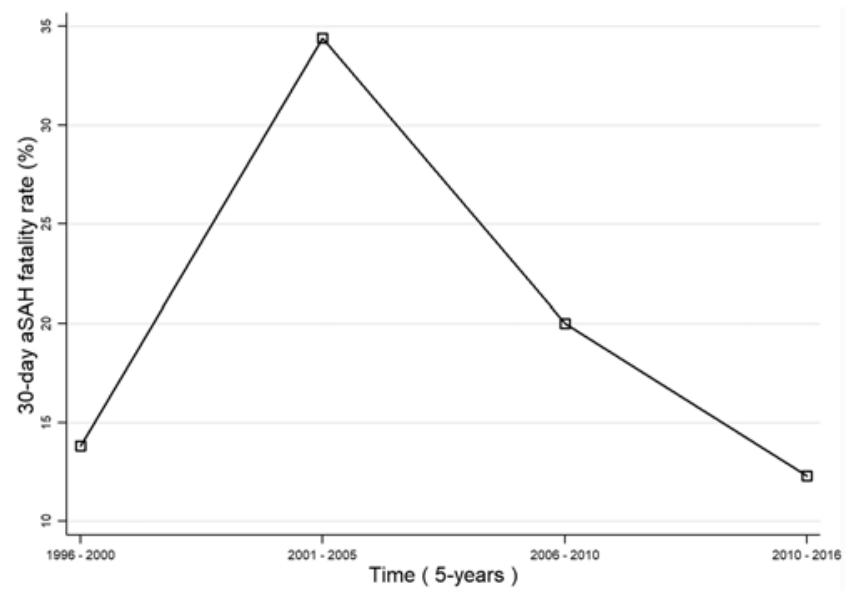

FIG. 2. Decline in 30 -day aSAH fatality rates by 5 -year intervals from 1996 to 2016. 
TABLE 4. Smoking frequency in patients with ulAs and ruptured aSAH in Olmsted County, Minnesota (1996-2016)

\begin{tabular}{lccccc}
\hline & \multicolumn{3}{c}{ Frequency of Smoking Patients by Decade } \\
\cline { 2 - 3 } & \multicolumn{2}{c}{ Patients w/ ulAs } & & \multicolumn{2}{c}{ Among aSAH Patients } \\
\cline { 2 - 3 } \cline { 5 - 6 } Smoking Status & $1996-2005$ & $2006-2015$ & & $1996-2005$ & $2006-2015$ \\
\hline Active smokers & $16 \%$ & $14.7 \%$ & & $47.3 \%$ & $42.3 \%$ \\
\hline Former smokers & $5.5 \%$ & $3.4 \%$ & & $17.5 \%$ & $17.2 \%$ \\
\hline Nonsmokers & $78.5 \%$ & $81.9 \%$ & & $35 \%$ & $40.5 \%$ \\
\hline
\end{tabular}

${ }^{*}$ Former $=\geq 12$ months.

aSAH was $22 \%$ over the past 2 decades. In a hospital-based study, we noticed similar trends with average in-hospital fatality rates decreasing from $22.6 \%$ in the period between 1985 and 1994 to $16.3 \%$ between 1995 and 2004, and then remaining relatively constant at $16.7 \%$ between 2005 and 2014. ${ }^{9}$ The reasons for better case-fatality rates are likely multifactorial and include advances in endovascular and surgical therapy, and improvement in multidisciplinary neurocritical care with better understanding and management of aSAH and its neurological and systemic complications. ${ }^{9}$

\section{Limitations}

The generalizability of these data to other populations is unclear given that the studied population differs somewhat from the US overall, including the proportion of minorities, level of education with a higher frequency of high school graduation, and frequency of income below the poverty level. ${ }^{14}$ However, our crude incidence rates were standardized to the male and female Caucasian North American population for each year from 1996 to 2016, to make our results more generalizable to the North American population, and potentially to the Western population. We also compared our data to worldwide trends in aSAH incidence and found that our results are consistent with those recently reported in the literature from other regions of the world. ${ }^{3}$ The interval of time studied was relatively short, but the study was specifically designed to exactly mirror a previous population-based study from Olmsted County (Menghini et al. ${ }^{12}$ ) to allow the analysis of aSAH incidence over the last 50 years in the same population. Case identification and data collection were performed using comprehensive medical records that included inpatient and outpatient data, discharge summaries, imaging reports, and autopsy data. Although data were checked several times, omissions or errors may have occurred; however, we have no reason to suspect a major misclassification bias. Given the retrospective nature of the data collection, the smoking information may have been impacted by several factors including accuracy of the historical data, and by recall bias at, or after, the aSAH. Some patients with hypertension may not have had a diagnosis reflected in the medical record, or they may have been taking an antihypertensive medication for some other purpose.

\section{Conclusions}

In our population-based study of a relatively homoge- neous North American population assessed from 1996 until 2016, we found the average incidence of aSAH to be $4.2 / 100,000$. The 30 -day case-fatality rate was $22 \%$. Both incidence and case-fatality rates showed a trend toward decreasing rates in this 20-year interval, which continued a similar trend previously noted in the same population.

\section{References}

1. Brown RD, Whisnant JP, Sicks JD, O'Fallon WM, Wiebers DO: Stroke incidence, prevalence, and survival: secular trends in Rochester, Minnesota, through 1989. Stroke 27:373-380, 1996

2. Cea Soriano L, Gaist D, Soriano-Gabarró M, Bromley S, García Rodríguez LA: Low-dose aspirin and risk of intracranial bleeds: an observational study in UK general practice. Neurology 89:2280-2287, 2017

3. Etminan N, Chang HS, Hackenberg K, de Rooij NK, Vergouwen MDI, Rinkel GJE, et al: Worldwide incidence of aneurysmal subarachnoid hemorrhage according to region, time period, blood pressure, and smoking prevalence in the population: a systematic review and meta-analysis. JAMA Neurol 76:588-597, 2019

4. Fisher CM, Kistler JP, Davis JM: Relation of cerebral vasospasm to subarachnoid hemorrhage visualized by computerized tomographic scanning. Neurosurgery 6:1-9, 1980

5. Giordan E, Brinjikji W, Vine RL, Lanzino G: Risk of de novo aneurysm formation in patients with unruptured intracranial aneurysms. Acta Neurochir (Wien) 160:747-751, 2018

6. Gupta PD: A general method of correction for age misreporting in census populations. Demography 12:303-312, 1975

7. Hasan DM, Mahaney KB, Brown RD Jr, Meissner I, Piepgras DG, Huston J, et al: Aspirin as a promising agent for decreasing incidence of cerebral aneurysm rupture. Stroke 42:3156-3162, 2011

8. Korja M, Lehto H, Juvela S, Kaprio J: Incidence of subarachnoid hemorrhage is decreasing together with decreasing smoking rates. Neurology 87:1118-1123, 2016

9. La Pira B, Singh TD, Rabinstein AA, Lanzino G: Time trends in outcomes after aneurysmal subarachnoid hemorrhage over the past 30 years. Mayo Clin Proc 93:1786-1793, 2018

10. Levy DT, Boyle RG, Abrams DB: The role of public policies in reducing smoking: the Minnesota SimSmoke tobacco policy model. Am J Prev Med 43 (5 Suppl 3):S179-S186, 2012

11. Menghini VV, Brown RDJ Jr, Sicks JD, O’Fallon WM, Wiebers DO: Clinical manifestations and survival rates among patients with saccular intracranial aneurysms: population-based study in Olmsted County, Minnesota, 1965 to 1995. Neurosurgery 49:251-258, 2001

12. Menghini VV, Brown RD Jr, Sicks JD, O'Fallon WM, Wiebers DO: Incidence and prevalence of intracranial aneurysms and hemorrhage in Olmsted County, Minnesota, 1965 to 1995. Neurology 51:405-411, 1998

13. Phillips LH II, Whisnant JP, O'Fallon WM, Sundt TM Jr: The unchanging pattern of subarachnoid hemorrhage in a community. Neurology 30:1034-1040, 1980

14. St Sauver JL, Grossardt BR, Leibson CL, Yawn BP, Melton LJ III, Rocca WA: Generalizability of epidemiological findings and public health decisions: an illustration from the Rochester Epidemiology Project. Mayo Clin Proc 87:151160, 2012

\section{Disclosures}

Dr. Lanzino reports having direct stock ownership in Marblehead and Superior Medical Editing. 
Giordan et al.

\section{Author Contributions}

Conception and design: Lanzino, Giordan. Acquisition of data: Giordan. Analysis and interpretation of data: Giordan, Brown, Rocca. Drafting the article: all authors. Critically revising the article: all authors. Reviewed submitted version of manuscript: Giordan, Graffeo, Rabinstein, Brown, Rocca, Chamberlain. Approved the final version of the manuscript on behalf of all authors: Lanzino. Statistical analysis: Giordan. Study supervision: Lanzino.

\section{Correspondence}

Giuseppe Lanzino: Mayo Clinic, Rochester, MN. lanzino.giuseppe @mayo.edu. 